\title{
Intratrachealer Tumor bei einem 16-jährigen Jungen: Extrem seltene Primärlokalisation eines B-Zell-Non-Hodgkin-Lymphomes
}

\author{
H. Kraußer \\ A. Skuballa
}

\author{
Endotracheal Neoplasm of a 16-year-old Boy: Extremely Rare Primary \\ Localisation of a B-cell Non-Hodgkin's Lymphoma
}

\section{Zusammenfassung}

Die Trachea ist im Gegenstatz zum Larynx oder den Bronchien selten primärer Sitz eines Tumors. Bei den sekundären Tracheamalignomen handelt es sich meist um infiltrierende Ösophagusund Schilddrüsenkarzinome oder um infiltrierende Lymphknotenmetastasen. Systemische Non-Hodgkin-Lymphome können ebenfalls den oberen Tracheobronchialtrakt befallen. Hier wird der seltene Fall eines extranodalen intratrachealen NonHodgkin-Lymphoms eines 16-jährigen Jungen beschrieben. Wegen des allmählichen und uncharakteristischen Krankheitsbeginnes wurde der Patient über einen Zeitraum von mehreren Wochen unter der Verdachtsdiagnose Asthma bronchiale behandelt. Schließlich hatte der 2,5 cm große Tumor zu einer erheblichen Trachealstenose geführt. Durch eine starre Bronchoskopie konnte schließlich die Diagnose gesichert und eine Therapie der bedrohlichen Situation durch Abtragung des Tumors erfolgen.

\section{Abstract}

Primary neoplasms of the trachea are rare, in contrast to the larynx and the bronchial tree. Most common types of secondary tracheal tumors are infiltrating esophageal and thyreoid cancers, or infiltrating lymphe node metastasis. Systemic non-Hodgkin's lymphomas may also involve the upper airway. Here we describe the rare case of a primary extranodal endotracheal nonHodgkin's lymphoma of a 16-year-old boy, who was presumed to have asthma and received antiasthmatic therapy for some weeks. The tumor, $2.5 \mathrm{~cm}$ in size, caused significant tracheal stenosis. Using rigid bronchoscopy, the difficult diagnosis was revealed and specific therapy initiated.

\section{Fallbeschreibung}

Seit Oktober 2000 klagte ein damals 16-jähriger Junge über rezidivierende Atembeschwerden. Der Patient wurde ambulant unter dem Verdacht auf Asthma bronchiale medikamentös behandelt. Bei der Spirometrie ergab sich eine kombinierte restriktive obstruktive Ventilationsstörung. Trotz hochdosierter Prednisolongaben mit inhalativen Bronchodilatatoren und Antibiotikatherapie kam es zu keiner Beschwerdebesserung.
Wegen progredientem Stridor erfolgte am 13.11. 2000 die stationäre Aufnahme des Patienten in einer anderen Klinik, wobei sich nunmehr in der Fluss-Volumen-Kurve deutliche Hinweise für eine extrathorakale Stenose fanden. Bei der flexiblen Bronchoskopie zeigte sich ein fast vollständiger Verschluss des Tracheallumens durch einen kugeligen Tumor (Abb.1).

Im CT des Thorax bestätigte sich die wandhaftende intratracheale Raumforderung von 2,5 cm Größe in einer Entfernung von $2 \mathrm{~cm}$ von der Hauptbifurkation (Abb. 2). Es fanden sich keine zer- 


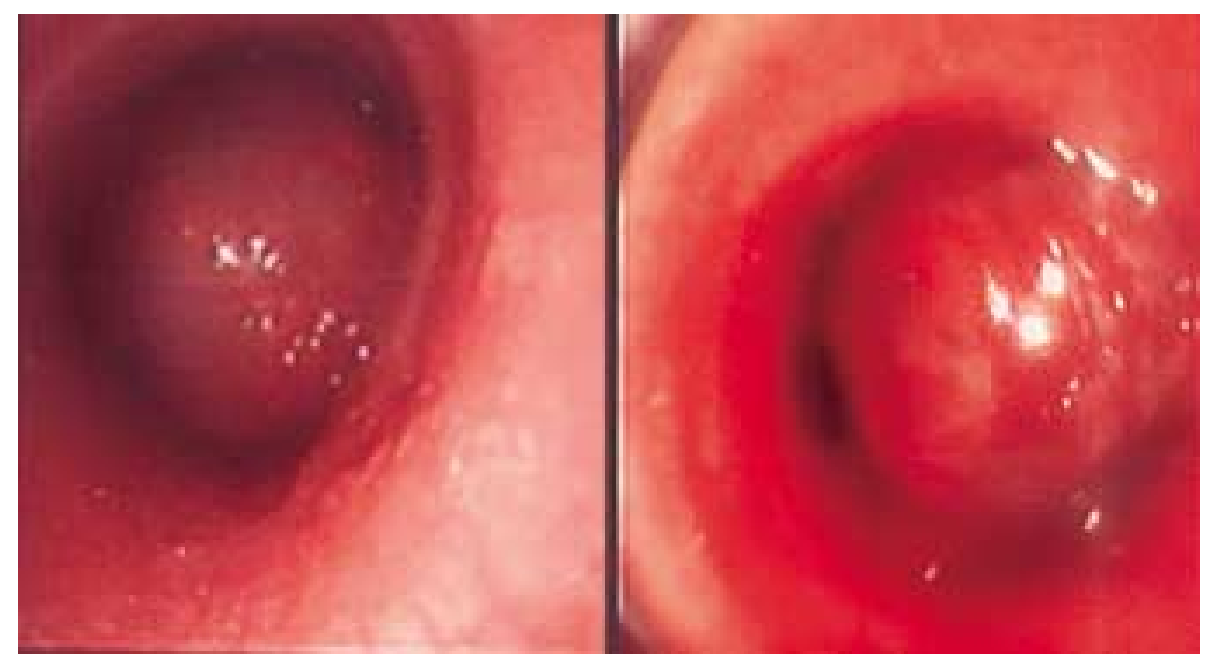

Abb. 1 Endotrachealer Tumor - Bronchoskopiebefund.

vikalen, mediastinalen oder hilären Lymphome. Schädel-CT und Sonographieuntersuchung des Abdomens waren ohne pathologischen Befund.

Wegen des fast vollständigen Tracheallumenverschlusses mit erheblichem Stridor erfolgte am 17.11. 2000 die Verlegung in unsere Klinik zur weiteren Intervention in Thorakotomiebereitschaft. Noch am Verlegungstag gelang es, durch eine starre Bronchoskopie den inzwischen bedrohlichen Zustand abzuwenden. Der endotracheale Tumor wurde zunächst durch Laserkoagulation (Nd: YAG-Laser, $1064 \mathrm{~nm}, 40 \mathrm{~W}$, gasgespülte Faser) verkleinert und anschließend mittels einer Diathermieschlinge (Fa. Storz, 60 W) abgetragen.

Durch die vollständige Tumorabtragung resultierte ein ca. $4 \mathrm{~mm}$ großer, der ehemaligen Tumorbasis entsprechender Defekt in der lateralen Trachealwand (Abb. 3). Um der Ausbildung eines progredienten Mediastinalemphysems respektive einer Mediastinitis vorzubeugen, erfolgte die Implantation eines PolyflexStents (Durchmesser $14 \mathrm{~mm}$, Länge $50 \mathrm{~mm}$ ) zur Abdichtung des Tracheallecks.

Der weitere Verlauf gestaltete sich komplikationslos. In den Folgetagen wurden wiederholt Bronchoskopien mit flexiblem Gerät zur Lagekontrolle und Prophylaxe einer Sekretretention durchgeführt. Ein initial durch Jet-Ventilation provoziertes Mediastinalemphysem war im weiteren Verlauf regredient.

Die histologische Aufarbeitung des resezierten endotrachealen Tumors gestaltete sich recht diffizil. Unter Einbeziehung des Konsultations- und Referenzzentrums für Lymphknotenpathologie in Würzburg wurde der endotracheale Tumor zusammenfassend als eine plasmoblastisch differenzierte und stark proliferationsaktive Neoplasie gedeutet, wobei differenzialdiagnostisch ein tumorbildendes extramedulläres anaplastisches Plasmozytom von einer plasmoblastischen Variante eines diffusen großzelligen B-Zell-Lymphoms zunächst nicht sicher unterschieden werden konnte (Abb.4).

Bei nunmehr völliger Beschwerdefreiheit wurde der Patient im Dezember 2000 zu ergänzenden Staginguntersuchungen erneut

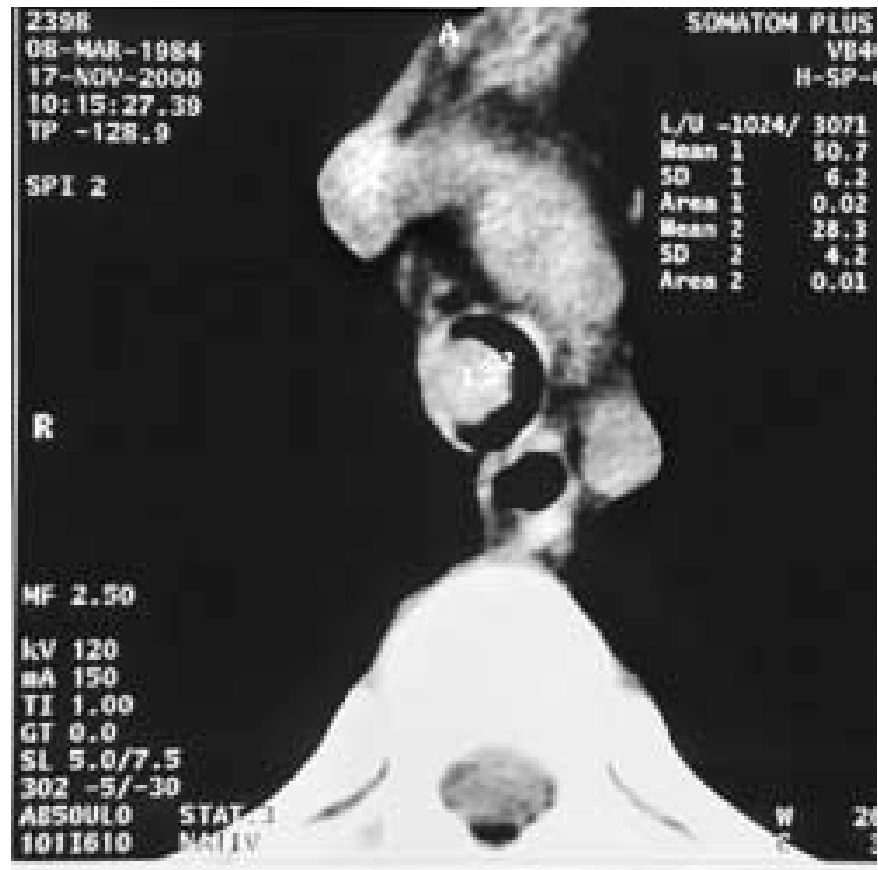

Abb. 2 CT-Befund des Tumors, von der rechten Trachealwand ausgehend. Die erhebliche Stenose der Trachea mit sichelförmigem Restlumen ist gut zu sehen.

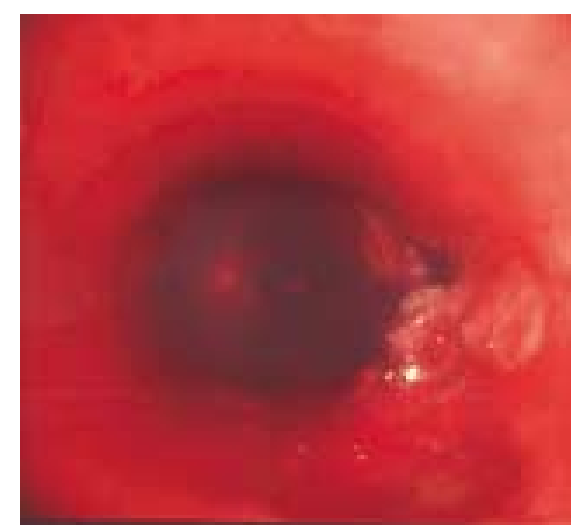

Abb. 3 Abtragungsstelle des Tumors. 


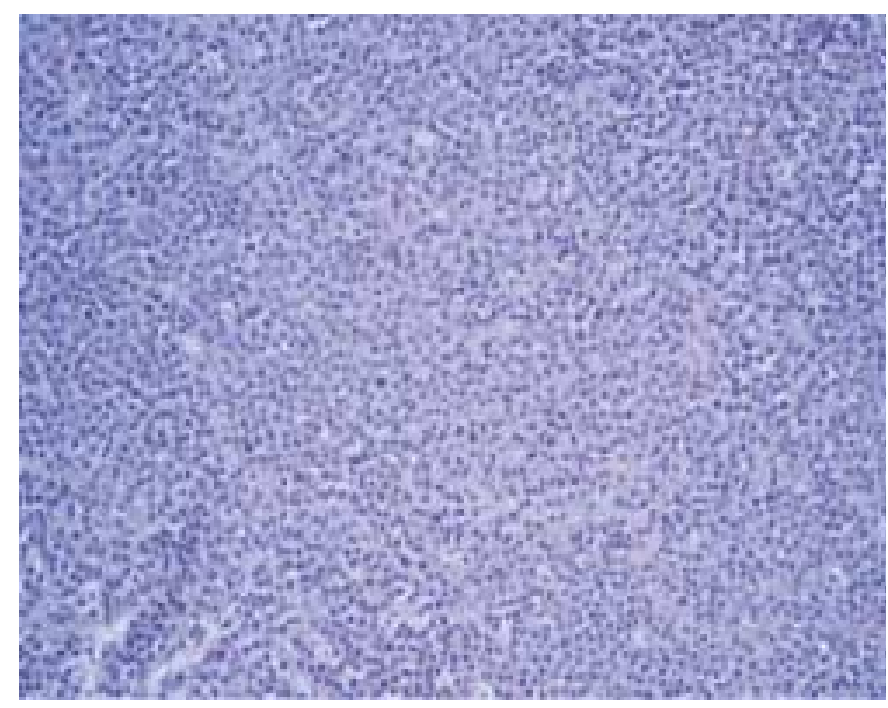

Abb. 4 Histologisches Präparat des Tumors, HE-Färbung.

stationär aufgenommen. Bei der CT-Kontrolluntersuchung fanden sich wiederum keine Hinweise auf abdominale oder intrathorakale Lymphome. Die Knochenmarkszytologie konnte keinerlei Anhalt für eine Infiltration durch ein B-Zell-Lymphom oder ein Plasmozytom erbringen. Es erfolgte die Verlegung in die Abteilung für pädiatrische Hämatologie und Onkologie der Universität Leipzig zur Initiierung einer protokollgerechten Chemotherapie.

Nach ergänzenden immunhistochemischen Untersuchungen wurde der Befund als eine Variante eines diffusen großzelligen B-Zell-Lymphomes eingestuft. Es wurde eine protokollgerechte Chemotherapie nach der NHL-BFM-95-Studie eingeleitet. Die Therapie, welche sich über einen Zeitraum von Dezember 2000 bis April 2001 erstreckte, hat der Patient relativ gut toleriert.

Nach dem ersten Chemotherapiezyklus expektorierte der Patient aufgrund der Tumorremission den Stent folgenlos. Bei der sofortigen Bronchoskopiekontrolle zeigte sich das Leck in der Trachealwand vollständig verschlossen, Tumorreste oder ein Rezidiv fanden sich nicht.

Am 6. 4. 2001 konnte der Patient endgültig in hausärztliche Betreuung entlassen werden. Die abschließende MRT-Untersuchung ergab keinen Hinweis auf ein erneutes Tumorgeschehen oder auffällige Lymphknoten. Der Patient stellt sich regelmäßig in der Dispensairesprechstunde in der Kinderklinik der Universität Leipzig vor. Er ist bisher rezidivfrei.

\section{Diskussion}

Die Trachea ist im Gegensatz zum Larynx oder den Bronchien selten primärer Sitz eines Tumors. Während bei Kindern ca. 90\% der Tracheatumoren benigne sind, überwiegen im Erwachsenenalter bei weitem maligne Tumoren und Tumoren mit niedrigem Malignitätsgrad [1,2]. Besonders bei Erwachsenen stellt das untere Drittel eine Prädilektionstelle für Tracheatumoren dar.
Unter den gutartigen mesenchymalen Tumoren sind die Fibrome, unter den gutartigen epithelialen die Adenome am häufigsten. Trachealpapillome entwickeln sich meist im Zusammenhang mit Larynxpapillomen.

Primär maligne Erkrankungen der Trachea sind sehr rar, sie haben etwa einen Anteil von 0,1\% aller Malignome. Unter den sehr seltenen Trachealkarzinomen überwiegt das Plattenepithelkarzinom [2-5].

Bei einer von Yang [5] durchgeführten Analyse von 67 Tracheamalignomen traten die Plattenepithelkarzinome am häufigsten auf mit einem Anteil von 52,2\% gefolgt von den Adenokarzinomen (14,9\%), den schlecht differenzierten Karzinomen (10,4\%) und den adenoid-zystischen Karzinomen (7,5\%). In dieser Serie fanden sich zwei Lymphome der Trachea (3\%).

Bei den sekundären Tracheamalignomen handelt es sich meist um infiltrierende Ösophagus-, Bronchial- und Schilddrüsenkarzinome oder um infiltrierende Lymphknotenmetastasen.

Systemische Lymphome können ebenfalls den oberen Tracheobronchialtrakt befallen. Freeman [6] beschrieb beispielsweise in einer Serie von 1467 Patienten mit einem extranodalen NonHodgkin-Lymphom 53 Patienten mit einem Befall der Lunge, des Tracheobronchialbaumes oder der Pleura. Fidias [3] fand bei einer Auswertung von 7 Patientengruppen mit insgesamt 425 Fällen eines Trachealtumors lediglich ein Non-Hodgkin-Lymphom $(0,23 \%)$.

Die Primärmanifestation eines extranodalen Non-Hodgkin-Lymphomes in der Trachea wie bei dem von uns vorgestellten Fall stellt eine absolute Rarität dar [1,3,7-12]. Gomez-Roman u. Mitarb. [7] beschrieben 1999 den ersten Fall eines extranodalen endotrachealen lymphoplasmozytoiden Lymphomes bei bis zu diesem Zeitpunkt insgesamt lediglich 9 in der Weltliteratur veröffentlichten Fällen einer Primärmanifestation eines NonHodgkin-Lymphomes in der Trachea.

Der lymphomatöse Tumor in den oberen Atemwegen führt meist zu uncharakteristischen Symptomen einer Obstruktion mit pfeifendem Atemgeräusch, Dyspnoe und in der Regel unproduktivem Husten. Daher wird die Erkrankung häufig wie in dem von uns beschriebenen Fall als Asthma bronchiale fehlgedeutet $[3,5,13]$. Hämoptysen treten eher selten auf, was sich durch die oft makroskopisch und histologisch intakte Bronchialschleimhaut erklärt [14]. Im weiteren Verlauf tritt dann jedoch ein Stridor auf, anfangs nur unter körperlicher Belastung oder Hyperventilation. Die Verdachtsdiagnose extrathorakaler Tumor kann durch die charakteristische Fluss-Volumen-Kurve gestellt werden. Auffällige Ergebnisse der Spirometrie, progrediente Dyspnoe und fehlender Behandlungserfolg indizieren häufig eine Bronchoskopie, welche zur richtigen Diagnose führt [7].

Bei malignen Lymphomen kann der mediastinale oder zervikale Lymphknotenbefall durch Kompression und Infiltration der zentralen Luftwege oder - wie in unserem Fall - durch das intramurale Tumorwachstum mit einer Lumenverlegung von mehr als $75 \%$ rasch zu einer akuten Atemnotsymptomatik führen. Die- 
se stellt eine Herausforderung für alle an der Behandlung der Patienten beteiligten Ärzte dar $[13,15,16]$.

Als besonders hilfreich erweisen sich in solchen Fällen die interventionellen Möglichkeiten einer starren Bronchoskopie mit ihren vielfältigen Optionen sowohl zur Diagnosesicherung durch Biopsie oder Punktion als auch zur Therapie z. B. Rekanalisierung durch Tumorabtragung mittels scharfer Instrumente, Laserbehandlung, Argon-Plasmakoagulation oder Elektrokoagulation.

Eine weitere Option der starren Bronchoskopie besteht in der Möglichkeit einer Stentimplantation in die Trachea. Die Intention der starren Bronchoskopie besteht dabei in der Behebung der Notfallsituation und der Überbrückung des erforderlichen Zeitraums bis zur erfolgreichen tumorspezifischen Therapie. Die Art des zu wählenden Stents hängt ab von der Lage und Ausdehnung der Stenose, der Prognose der Erkrankung und der tumorspezifischen Therapie $[4,13,17-19]$.

Auch die Folgezustände einer endotrachealen Tumorabtragung wie beispielsweise Trachealwandinstabilitäten oder Trachealwandleckagen können mittels verschiedener Stentimplantationssysteme in gleicher Sitzung beherrscht werden. Regelmäßige Kontrollbronchoskopien sind zur Lagekontrolle und Beurteilung des Stenosegrades erforderlich $[16,19,20]$.

Der beschriebene Fall veranschaulicht deutlich, dass eine adäquate onkologische Behandlung meist eine interdisziplinäre $\mathrm{Zu}$ sammenarbeit verschiedener Fachgebiete voraussetzt. Auf diese Art und Weise ist es uns erfolgreich gelungen, eine tumorbedingte akute Atemnotsituation durch den Einsatz der interventionellen Bronchologie abzuwenden sowie durch erste therapeutische Maßnahmen genügend Freiraum für weitere umfassende Diagnostik zu schaffen. Auf deren Grundlage konnte der Patient schließlich einer adäquaten stadiengerechten onkologischen Behandlung im Rahmen einer Studie zugeführt werden.

\section{Literatur}

${ }^{1}$ Houston HE, Payne WS, Harrison EG et al. Primary cancers of the trachea. Arch Surg 1969; 99: 132-139

${ }^{2}$ Schildberg FW, Meyer G. Erkrankungen der Trachea und Hauptbronchien. In: Heberer G, Schildberg FW, Sunder-Plassmann L, Vogt-Moykopf I (Hrsg): Lunge und Mediastinum. Berlin, Heidelberg, New York: Springer, 1991: $292-295$

${ }^{3}$ Fidias P, Wright C, Harris NL et al. Primary tracheal Non-Hodgkin's lymphoma. A case report and review of the literature. Cancer 1996; 77 (11): $2332-2338$

${ }^{4}$ Grillo HC, Mathisen DJ, Wain JC. Management of tumors of the trachea. Oncology 1992; 6: 61-67

${ }^{5}$ Yang KY, Chen YM, Huang MH et al. Revisit of primary malign neoplasms of the trachea: clinical characteristics and survival analysis. Jpn J Clin Oncol 1997; 27 (5): 305 - 309

${ }^{6}$ Freeman C, Berg JW, Cutler SJ. Occurrance and prognosis of extranodal lymphomas. Cancer 1972; 29: $252-260$

${ }^{7}$ Gomez-Roman JJ, Perez-Montes R, Perez-Exposito MA et al. Primary lymphoplasmocytoid lymphoma of the trachea with immunoglobulin G paraprotein. Pathol Int 1999; 49 (12): 1100-1104

${ }^{8}$ Kaplan MA, Pettit CL, Zukerberg LR et al. Primary lymphoma of the trachea with morphologic and immunophenotypic characteristics of low-grade B-cell lymphoma of mucosa-associated lymphoid tissue. Am J Surg Path 1992; 16: 71-75

${ }^{9}$ Maeda M, Kotake Y, Monden Y et al. Primary malignant lymphoma of the trachea: report of a case successfully treated by primary end to end anastomosis after circumferential resection of the trachea. J Thorac Cardiovasc Surg 1981; 81: 835-839

${ }^{10}$ Pradhan DJ, Rabuzzi D, Meyer JA. Primary solitary lymphoma of the trachea. J Thorac Cardiovasc Surg 1975; 70: 938 -940

${ }^{11}$ Spapen H, Eeckhout E, Volckaert A et al. Primary endobronchial NonHodgkin's lymphoma: report of two cases and review of the literature. Neth J Med 1988; 33: 86-90

12 Wiggins J, Sheffield E, Green M. Primary B cell malignant lymphoma of the trachea. Thorax 1988; 43: 497-498

${ }^{13}$ Schmidt B, Massenkeil G, John M et al. Temporary tracheobronchial stenting in malignant lymphoma. Ann Thorac Surg 1999; 67 (5): $1448-1450$

${ }^{14}$ Schrijver M, Havenith MG, Hupperets PSGJ et al. Endobronchial NonHodgkin's-Lymphoma: report of two cases. Respiration 1987; 2: $228-231$

${ }^{15}$ Eng J, Sabanathan S. Endobronchial Non-Hodgkin's lymphoma. J Cardiovasc Surg 1993; 34: 351 - 354

${ }^{16}$ Unger M. Tracheobronchial stents and stunts. Chest 1990; 97: $260-261$

${ }^{17}$ Fridel G, Wurst H, Hürtgen $M$ et al. Die endoluminale Therapie in Trachea und Bronchus. Chirurg 2001; 72: 1119-1129

${ }^{18}$ Shah R, Sabanathan S, Mearns AJ et al. Self-expanding tracheobronchial stents in the management of major airway problems. J Cardiovasc Surg 1995; 36 (4): $343-348$

${ }^{19}$ Deutsche Gesellschaft für Pneumologie, Arbeitsgruppe „Qualitätssicherung in der Bronchologie“. Empfehlungen zur bronchoskopischen Behandlung tracheobronchialer Verschlüsse, Stenosen und muraler maligner Tumoren. Pneumologie 1998; 52: 243 - 248

${ }^{20}$ Witt C, Dinges S, Schmidt B et al. Temporary tracheobronchial stenting in malignant stenoses. Eur J Cancer 1997; 33: 204 - 208 